\section{MS27-P7 Dynamically refined PEDT of Ni2Si compared to XRD}

Cinthia A. Corrêa ${ }^{1,2}$, Mariana Klementová ${ }^{2}$, Lukáš Palatinus ${ }^{2}$

1. Department of Physics of Materials, Charles University Prague

2. Institute of Physics, Academy of Sciences of the Czech Republic

email: cinthiacac@gmail.com

Electron diffraction tomography (EDT) [1] combined with the precession electron diffraction (PED) [2] are ideal techniques for structural analysis of single nanocrystals. The strong interaction between electrons and matter, compared to X-ray, allows structural analysis of nanocrystals as small as tens of nanometers. During the EDT experiment, the crystal is tilted in small steps and a diffraction pattern is collected for each position, giving three-dimensional information of the reciprocal space. However, the strong interaction between the electron beam and matter generates multiple scattering of the beam and the accurate structure refinement requires the use of the dynamical diffraction theory for the calculation of model intensities. The dynamical character of the reflections can be suppressed by using PED. The technique consists of precessing the beam around the optical axis of the microscope, where the resulting intensities are integrated for each angular position of the beam. These integrated intensities are more sensitive to structure parameters and less sensitive to crystal imperfections. Despite of the damping of the dynamical effects, the refinement against precession EDT (PEDT) data using kinematical diffraction theory gives low accuracy of the structure parameters and high residual factors. The dynamical refinement for EDT data sets was implemented recently [3] and has been shown to be superior to the kinematical refinement, giving lower figures of merit and higher accuracy of the structure parameters. In this work, the dynamical refinement is used for the structure analysis of a nanowire of $\mathrm{Ni}_{2} \mathrm{Si}$ with the diameter of $16 \mathrm{~nm}$. The dynamical refinement against a data set collected by PEDT is compared to kinematical refinement and to a reference structure, obtained by single crystal X-ray diffraction. The structure has orthorhombic Pnma symmetry with 3 independent atoms. It is shown that the single crystal X-ray structure and the PEDT structure match very well with an average distance of atomic positions less than $0.015 \AA$.

[1] Kolb, U., Gorelik, T., Kuebel, C., Otten, M.T., Hubert, D. (2007). Ultramicroscopy 107 (6-7), 507-513

[2] Vincent, R., Midgley, P.A. (1994). Ultramicroscopy 53 (3), 271-282. Mugnaioli, E., Gorelik, T., Kolb, U. (2009). Ultramicroscopy 109 (6), 758-765

[3] Palatinus, L., Petř́íček, V., Corrêa, C.A. (2015). Acta Crystallographica A 71, 235-244

This work is financially supported by the Czech Science Foundation, project 13-25747S.

Keywords: Nanocrystal, precession electron diffraction tomography, dynamical refinement, X-ray.

\section{MS27-P8 Machine learning decomposition} of scanning precession electron diffraction data

Duncan N. Johnstone ${ }^{1}$, Alexander S. Eggeman ${ }^{1}$, Paul A. Midgley ${ }^{1}$

1. Department of Materials Science \& Metallurgy, University of Cambridge, UK

email:dnj23@cam.ac.uk

Scanning precession electron diffraction (SPED) is being developed to map the crystalline phases in materials with $\mathrm{nm}$ resolution and across micron sized regions. Precession electron diffraction (PED) patterns are recorded using a double conical rocking beam system [1] as the electron beam is scanned across the sample [2]. The 4-dimensional (two real \& two reciprocal) data set can be analysed in a number of ways. Most simply, 'virtual' dark-field images can be formed by plotting the intensity of a diffracted beam (sub-set of pixels) as a function of beam position. Each PED pattern can also be matched to a library of simulated patterns, determining the orientation and producing orientation images, typically as Euler angle maps [2]. This analysis is hindered by 'artefacts' such as specimen bending and thickness variations, the effect of which can be reduced by utilising larger precession angles. The remaining challenge that we address is the appropriate treatment of diffraction patterns that comprise signals from overlapping crystals.

We apply machine learning approaches based on non-negative matrix factorisation (NMF) and principal component analysis (PCA) combined with independent component analysis (ICA) in order to learn the underlying 'component patterns', that make up the data, together with their associated 'loadings' at each real space pixel. An example of such decomposition is shown in the figure below. Loading maps indicate the region where the component pattern is significant and resembles a simplified dark-field image. Loading maps corresponding to different component patterns may have overlapping regions of intensity where mixed diffraction patterns have been separated into individual components. This decomposition is the key to performing 3D volume reconstructions with crystallographic information at each voxel [3]. Here we report on the development of this approach by evaluating the decomposition of PED data systematically using a number of algorithms. In particular, results are presented demonstrating the merits of sparsity and non-negativity constraints imposed on the aforementioned decompositions. Twin boundaries in GaAs nanowires were used as a test case to validate the methods, which have then been applied to a number of more complex cases.

[1] R Vincent, and P A Midgley, Ultramicroscopy, $1994,53,271-282$

[2] E Rauch et al, Zeitshrift fur Kristallographie 2010, 225, 103-109

[3] A S Eggeman, R Krakow, and P A Midgley, submitted 